\title{
Pediatric RCVS in Newly Diagnosed all Treated with Intra- Arterial Nicardipine
}

\author{
Devin Simon, $M D^{1^{*}}$, Jaclyn Peraino, $B S^{2}$, Kabelo Thusang, $M D^{1}$ and Suhad Khalil, $M D^{1}$ \\ ${ }^{1}$ Department of Neurology and Ophthalmology, Sparrow Hospital, Michigan State University, USA \\ ${ }^{2}$ College of Osteopathic Medicine, Michigan State University, USA
}

\begin{abstract}
Background: Reversible Cerebral Vasoconstriction Syndrome (RCVS) is an uncommon cause of severe headache and other complications in the pediatric population. Diagnosis includes obtaining vessel imaging to confirm vasospasm in the cerebral vasculature. Therapy oftentimes requires calcium channel blockers administered through an intra-arterial approach. Resolution of symptoms and vasospasm are expected at approximately 3 months.

Case description: We report a case of RCVS in a 3-year-old male who was admitted to the pediatric ICU with newly diagnosed Acute Lymphoblastic Leukemia (ALL). Hospital course was complicated by acute neurologic decline and evidence of severe cerebral vasospasm resulting in multiple ischemic strokes. Course of treatment required hospital transfer for intra-arterial calcium channel blocker therapy with eventual resolution of cerebrovascular constriction and improvement in clinical exam.
\end{abstract}

Conclusion: When pediatric patients develop acute onset severe headaches, consideration of a broad differential diagnosis is important, and recognition of RCVS early in the hospital course can profoundly alter management and treatment options.

Keywords

Pediatrics, Acute Lymphoblastic Leukemia, RCVS, Stroke, Nicardipine

\section{Introduction}

Reversible Cerebral Vasoconstriction Syndrome (RCVS) is a rare disorder causing diffuse, severe headache of abrupt or progressive onset, with/without focal neurological deficits and/or seizures, accompanied by cerebral vasospasm, and often resolving within 12 weeks [1]. The mechanism is thought to involve dysregulation of cerebral vascular tone secondary to sympathetic overactivity. Median age of onset in females is around 50 , while slightly younger in males. There have only been a handful of pediatric cases described in the medical literature [2-4], and to our knowledge we are reporting the youngest case of RCVS in a male patient who had a successful neurologic outcome following intra-arterial calcium channel antagonism.

\section{Clinical Presentation}

A previously healthy 3-year-old male presented to the hospital due to worsening fatigue and bloodwork, concerning for pancytopenia with blast cells. Hematology/Oncology were consulted and diagnosed him with Acute Lymphoblastic Leukemia (ALL). He was admitted to the Pediatric ICU and chemotherapy was initiated.

He developed a headache and mild hypertension early in admission, with evidence of minor subarachnoid hemorrhage on CT imaging. Neurosurgery and Neurology assessments were requested, with recommendation to add anti-seizure medication as prophylaxis. 2 hour EEG was normal, and repeated CT head imaging was stable.

Additional imaging was obtained for further characterization of the subarachnoid hemorrhage which appeared atypical. MRA Head demonstrated patent intracranial arterial vessels without obvious aneurysm, however an underlying AVM couldn't be excluded (Figure 1). CTA Head/Neck with venous phase was recommended to evaluate for potential bleeding source.

*Corresponding author: Devin Simon, MD, Department of Neurology and Ophthalmology, Sparrow Hospital, Michigan State University, 804 Service Road, Lansing, MI 48824, Michigan, USA, Tel: 810-357-2726

Accepted: August 25, 2020

Published online: August 27, 2020

Citation: Simon D, Peraino J, Thusang K, et al. (2020) Pediatric RCVS in Newly Diagnosed all Treated with Intra-Arterial Nicardipine. J Pediatr Neurol Neurosci 4(1):76-78 

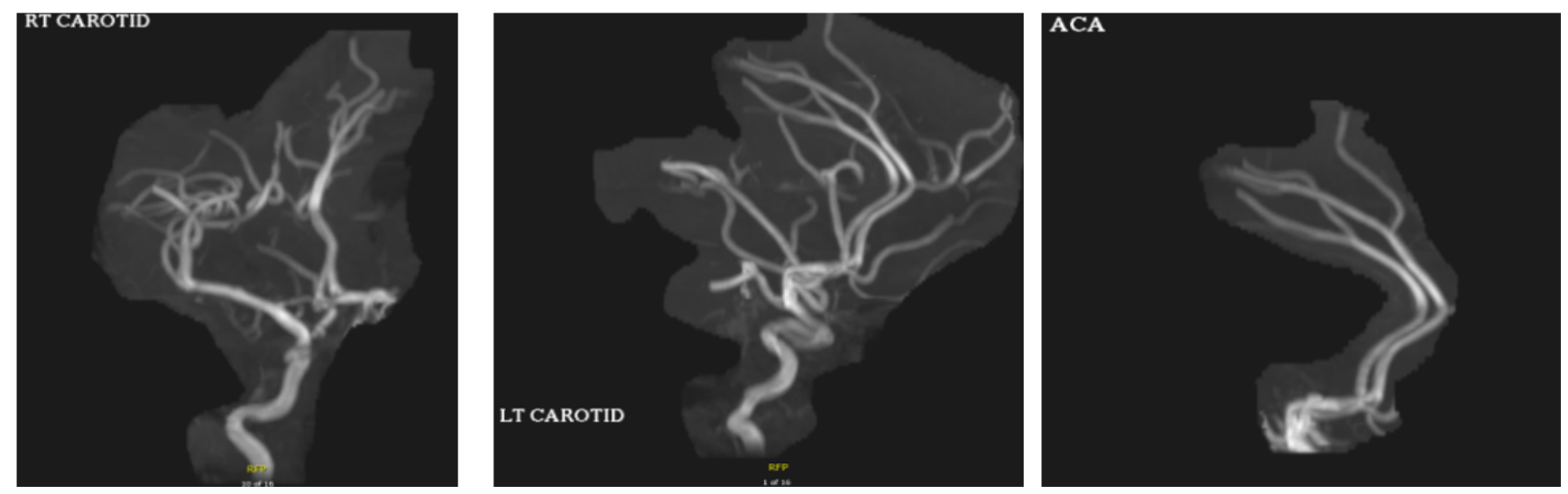

Figure 1: Initial MRA Head showing patent intracranial arterial vessels.
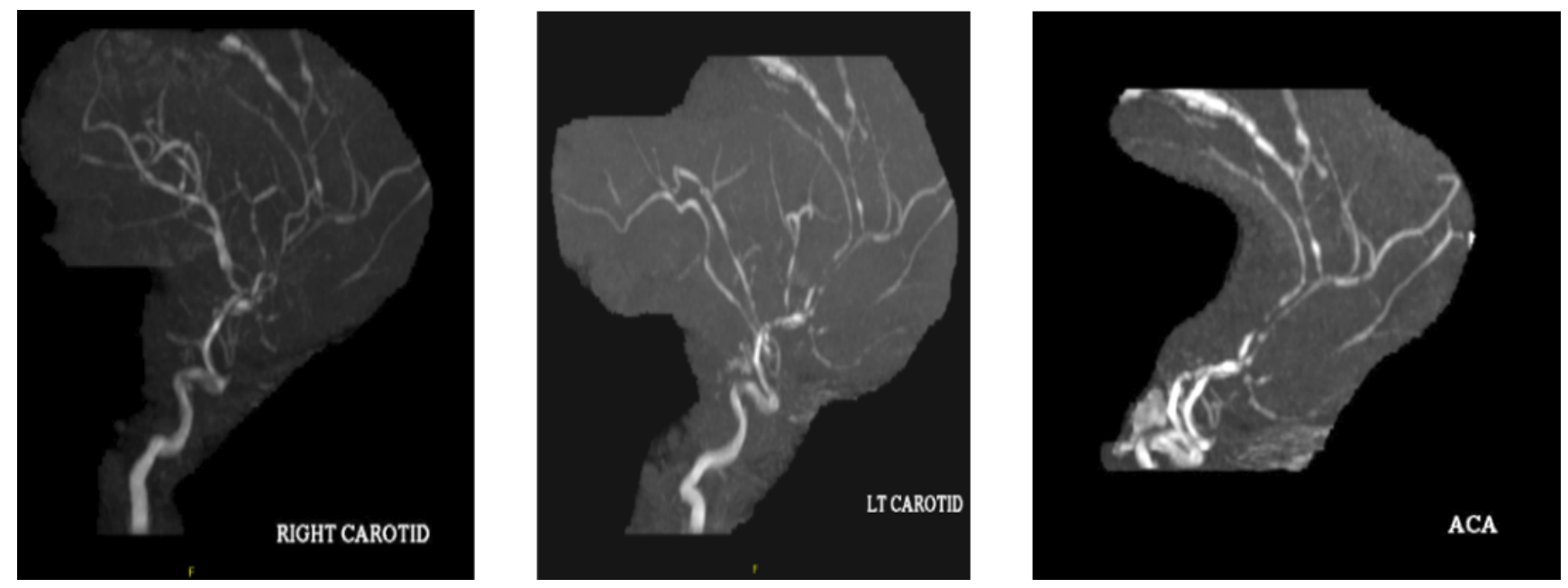

Figure 2: Repeat MRA Head imaging with evidence of diffuse luminal narrowing and areas of stricture/beading of multiple vessels.

There was difficulty controlling his blood pressure which fluctuated dramatically. Shortly after initiating chemotherapy, he experienced sudden onset exploding headache, with repeat CT angiogram showing multifocal segmental vascular beading and stenosis suggesting RCVS. Nicardipine drip was initiated in addition to oral antihypertensives for blood pressure control. Norvasc was chosen instead of Verapamil given persistent bradycardia and findings of reduced ejection fraction on echocardiogram. Repeat examination was concerning for pronounced right leg weakness and difficulty ambulating which were new findings. MRI Brain showed multifocal cortical strokes. MRA Head showed significant interval change since prior study, with diffuse luminal narrowing of multiple vessels throughout the anterior and posterior circulation (Figure 2).

He required transfer to a tertiary center for a therapeutic cerebral angiogram. Following worsening of his exam, additional imaging was significant for new ischemic strokes. Intraoperative imaging was consistent with diffuse cerebral vasospasms, and intra-arterial Nicardipine was administered. Despite brief stabilization for several days, he required another angiogram procedure with repeat injection of Nicardipine resulting in eventual improvement. He was stabilized with subsequent transfer back to our facility to continue chemotherapy.

Repeat MRA Head imaging 11 weeks later showed resolution of previous cerebral vasospasms and he had significant recovery of his previous symptoms.

\section{Discussion}

The differential diagnosis for pediatric headaches should include subarachnoid hemorrhage, venous thrombosis, unruptured intracranial aneurysm, artery dissection, hypertensive crisis, pituitary apoplexy, posterior reversible encephalopathy and RCVS. ALL is a common childhood malignancy and although treatment can be successful. There are always risks of side effects. RCVS has been associated with chemotherapy previously and needs to be considered if a pediatric patient suddenly becomes symptomatic with focal neurologic deficits [5]. There have been 4 pediatric cases of severe cerebral vasospasm with subsequent ischemic stroke in the setting of IT cytarabine therapy, which possibly suggests a link [6]. It is unclear if PEG-ASP was the sole risk factor for development of RCVS, as our patient already had evidence of mild subarachnoid hemorrhage on head imaging. Diagnosing RCVS can also be difficult as some cases in the pediatric population 
have occurred without headache. But, presentation with other neurologic symptoms such as seizures [7].

To our knowledge this is the youngest male described in the medical literature with RCVS. Early recognition can profoundly alter management and treatment options. Although uncommon, this should be included in the differential for patients undergoing chemotherapy or who have abnormal brain imaging with acute neurologic symptoms.

\section{Author Disclosure}

Drs. Simon, Thusang, Khalil, and Ms. Peraino have disclosed no financial relationships relevant to this article. This commentary does not contain a discussion of an unapproved/ investigative use of a commercial product/device.

\section{References}

1. Chen SP, Fuh JL, Wang SJ (2010) Reversible cerebral vasoconstriction syndrome: An under-recognized clinical emergency. Ther Adv Neurol Disord 3: 161-171.

2. Oikawa Y, Okubo Y, Numata-Uematsu Y, et al. (2017) Initial vaso- dilation in a child with reversible cerebral vasoconstriction syndrome. J Clin Neurosci 39: 108-110.

3. Kayfan S, Sharifi A, Xie S, et al. (2019) MRA and ASL perfusion findings in pediatric reversible cerebral vasoconstriction syndrome. Radiology Case Reports 14: 832-836.

4. Kazato Y, Fujii K, Oba H, et al. (2012) Reversible cerebral vasoconstriction syndrome associated with brain parenchymal hemorrhage. Brain Dev 34: 696-699.

5. Pound C, Keene D, Udjus K, et al. (2007) Acute encephalopathy and cerebral vasospasm after multiagent chemotherapy including PEG-asparaginase and intrathecal cytarabine for the treatment of acute lymphoblastic leukemia. J Pediatr Hematol Oncol 29: 183-186.

6. Tibussek D, Natesirinilkul R, Sun L, et al. (2016) Severe cerebral vasospasm and childhood arterial ischemic stroke after intrathecal cytarabine. Pediatrics 137: e20152143.

7. Kuga S, Goto H, Okanari K, et al. (2016) Reversible cerebral vasoconstriction syndrome manifesting as focal seizures without a thunderclap headache: A Pediatric case report. Brain and Development 38: 880-883.

DOI: $10.36959 / 595 / 411$ 